\title{
Ultrasonic Particle Velocimetry In Multiphase Flows
}

\author{
J. Carlson ${ }^{\dagger, *}$ and R. K. Ing ${ }^{\ddagger}$ \\ ${ }^{\dagger}$ EISLAB, Dept. of Computer Science and Electrical Engineering, Luleå University of Technology, \\ SE-971 87 Luleå, SWEDEN \\ $\ddagger$ Laboratoire Ondes et Acoustique, ESPCI - 10 rue Vauquelin, FR-75231 Paris Cedex 05, FRANCE. \\ *E-mail: Johan.Carlson@sm.luth.se
}

\begin{abstract}
Two-dimensional ultrasonic speckle correlation velocimetry (USV) is a new technique that allows to image moving scattering media, at a high frame-rate. In this paper we apply the technique to determine two-dimensional particle velocity profiles of multiphase flows. Experiments are realized with suspensions of Sonazoid ${ }^{1}$ (medical contrast agent) and Magnetite $\left(\mathrm{Fe}_{3} \mathrm{O}_{4}\right)$ in water. All measurements are performed in a vertical pipe with the flow moving downwards. The twodimensional particle velocity profiles are then compared with a reference liquid volume flow velocity. As expected from theory, the heavier Magnetite particles have slightly higher velocity than the liquid whereas the contrast agent simply follows the liquid motion.

The proposed technique can be used in combination with other techniques to measure the mass flow of the solid phase, in solid/liquid multiphase flow. This is generally more interesting than measuring the bulk mass or volume flow.
\end{abstract}

\section{INTRODUCTION}

During the last ten to fifteen years the measurement of different properties of multiphase flows has received a lot of attention in the flow measurement community. In some industrial processes, such as the mining industry, a liquid flow is used to transport solid particles. In such flows, the major interest lies in estimating the amount of particles transported, and not to measure the bulk flow itself. Ultrasound provides a direct way of global and non-invasive measurement of several parameters of such flows. For single phase flows of liquids and gases there are several wellestablished ultrasonic techniques available based on the differences in transit-time for sound propagating upstream and downstream in the flow. In the presence of particles, the sound is scattered and the waveform of the sound pulse is heavily distorted. Because of this, determining transittimes becomes difficult, and therefore these techniques are not suitable for solid/liquid multiphase flows. Another technique consists of using the pulsed Ultrasonic Doppler Velocimetry (UDV). The term Doppler is unfortunately a misleading name for such a technique, because instead of measuring the actual Doppler shift, the velocity is determined by

\footnotetext{
${ }^{1}$ Sonazoid is a registered trademark of Nycomed Amersham A/S.
}

estimating the time delay between two backscattered pulses resulting from two consecutive transmissions. Contrarily to the transit-time techniques, the use of pulsed UDV is limited to applications where the medium contains scatterers. Still, cross-correlation based methods are preferred over transittime techniques. The most important difference for this is that transit-time techniques measure the bulk flow velocity, while cross-correlation techniques measure the motion of the scatterers in the medium. The UDV is generally used to determine the particle displacements along the longitudinal axis of pre-focused transducers. It is however also applied to sequential sectorial scan ultrasonic imaging systems usually found in the medical area to two dimensionally measure the flow velocity in blood vessels. In this case, the biological cell displacements are extracted from all the line signals that compose sectorial scan images. In the human body, the UDV still functions because the flow rate in blood vessels is low enough to ensure that two sequential line signals of two consecutive sectorial scan images - that are generally taken at a frame rate of $50 \mathrm{~Hz}$ or $60 \mathrm{~Hz}$ - are still correlated.

In this paper, the two dimensional ultrasonic speckle correlation velocimetry (USV) [1] technique is used to measure the particle velocities directly over the entire 2D crosssection of the flow pipe. Such a technique is associated with an ultrafast ultrasonic imaging system that increases the frame rate of ultrasonic images and allows us to measure high flow rates. The USV technique is similar to the UDV technique in the sense that it uses cross-correlation process to extract the time delay, which in turn gives the particle displacement and velocity. In the USV technique case, the ultrasonic image exploited is not however obtained sequentially but in only one snapshot as, explained in section II. The USV technique is especially interesting in the case when the flow pipe is not axi-symmetric or when the flow profile is not axi-symmetric, i.e. when a $2 \mathrm{D}$ knowledge of the flow velocity is required. Furthermore, the USV technique is a useful complement to conventional laser-doppler or other optical techniques, since it does not require the flow to be transparent. 
For applications where the goal is to measure the mass flow of the solid phase, this technique can be combined with some other method that measures the mass fraction of the solid phase. One example of such a technique is based on the attenuation of pulsed ultrasound [2]. An overview of other techniques can be found in [3].

The USV technique presented here has previously been used in other applications to track particles, or to image vortices in a flow, see for example [1], [4], [5]. In some of these cases, the flow was seeded with a medical contrast agent with density close to that of the liquid. Because of this, tracking the motion of the particles should be a reasonable approximation of the motion of the liquid. In the case of solid/liquid multiphase flows, the use of a contrast agent is not necessary, since the scatterers are naturally present in the flow.

\section{EXPERIMENTS}

\section{A. Measurement Principle}

To track the particle motion in the flow, we use an array of ultrasound transducers. The array is first used to transmit a short pulse simultaneously on all elements, and is then used as receiver to record the backscattered signals. The medium is then assumed to be illuminated with a planar acoustic wave. The resulting B-SCAN signal is then converted to an image of the target by using a dynamic focusing process. This consists of delaying and summing parts of the B-SCAN signal corresponding to a certain distance from each of the array elements. For example, if the emitted plane wave is backscattered by a thin wire target, this will be represented by a curved wave front in the B-SCAN signal. After beamforming we instead obtain an image of the target itself. This is similar to scanning the target region by sequentially focusing the array in transmit mode for different angles and different depths. The most important difference is that in this case we obtain the final image after only one transmission. This enables us to image very fast moving phenomena, where a traditional sequential scan would give erroneous results. With the ultrafast imaging system we can record approximately $5000 \mathrm{~B}-\mathrm{SCAN}$ signals per second.

By acquiring two B-SCAN signals closely spaced in time and then cross-correlating the resulting speckle images linewise along the longitudinal axis, we can follow how groups of scatterers move. Each line of the beamformed images are divided into short segments and cross-correlated with the corresponding line of the next image. The time delay corresponding to the maximum of the cross-correlation gives the displacement of that segment of the image. To increase the resolution of the image further, we interpolate the crosscorrelation function around its maximum, using a simple parabolic function. We can thus observe image displace-

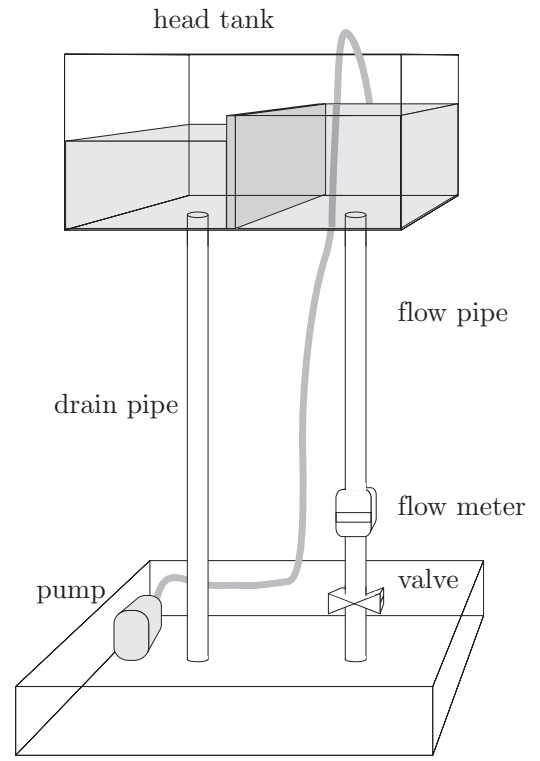

Fig. 1. Setup used in the experiments. The barrier in the head tank ensures constant pressure in the flow pipe. The flow velocity is controlled by the valve at the bottom of the pipe.

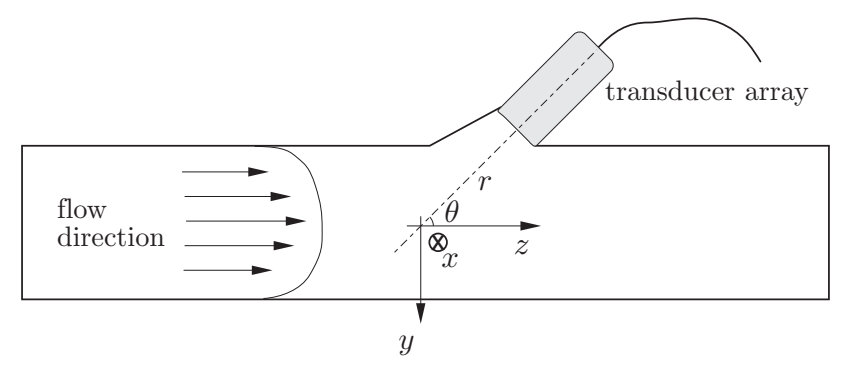

Fig. 2. The transducer array mounted with an incident angle of $45^{\circ}$. The $x$-direction in the figure is orthogonal to the $(z, y)$ plane.

ments of less than one sample.

\section{B. Setup}

For the experiments we used a 64 element transducer array with a center frequency of $3.5 \mathrm{MHz}$ and with a $0.417 \mathrm{~mm}$ element-pitch. The array was mounted perpendicular to the flow, with an incident angle of $45^{\circ}$ (see figures 1 and 2). The flow pipe was vertical, to ensure that even for large density ratios between the solid and liquid phases, the sedimentation would be minimal. The flow meter was mounted after one meter of straight pipe, in order to have a fully developed flow profile. The flow pipe was made of PVC plastic. The inner pipe diameter was $33.7 \mathrm{~mm}$.

From the transducer incident angle, $\theta=45^{\circ}$, and the particle displacement along the longitudinal axis, $r$, of the transducer array, the particle velocity, $v_{x}$, in the flow direction is given by

$$
v_{x}=v_{r} \cdot \sqrt{2} .
$$

To evaluate the technique we prepared two different sus- 
(a)

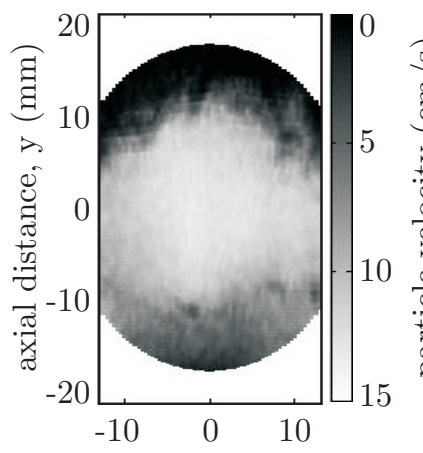

transversal distance, $\mathrm{x}(\mathrm{mm})$ (b)

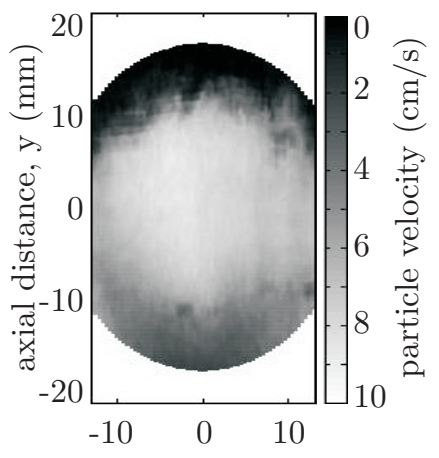

transversal distance, $\mathrm{x}(\mathrm{mm})$

Fig. 3. Sonazoid particles velocity profiles for entire cross section of the flow. (a) volume flow velocity $=8.96 \mathrm{~cm} / \mathrm{s}$. (b) volume flow velocity $=5.72 \mathrm{~cm} / \mathrm{s}$. The array was located at the bottom of the images.

pensions, with different density ratios between the solid and the liquid phase. The first suspension was water and the medical contrast agent Sonazoid. This contrast agent consist of small micro bubbles with approximately the same density as water. The second suspension was Magnetite $\left(\mathrm{Fe}_{3} \mathrm{O}_{4}\right)$ particles and water. The density of the Magnetite was 5200 $\mathrm{kg} / \mathrm{m}^{3}$, which is significantly higher than that of water.

\section{Results}

Figures 3 and 5 show the particle velocity cross sections of Sonazoid and Magnetite, respectively, for two different velocities. Figures 4 and 6 show the velocity profiles at the center transducer element of the array, for different velocities. A hole was cut in the wall of the pipe at the position where the transducer array was mounted. At this position small vortices formed, which disturbed the otherwise axisymmetric flow profile. This can be seen at the bottom of figures 3 and 5, but even clearer on the left side of figures 4 and 6.

For each of the experiments a measurement of the liquid volume flow was also made. The liquid volume flow velocities were compared with the average particle velocities, taken over the whole flow profiles in figures 3 and 5 . The volume flow was determined by measuring the amount of liquid that falls through the right pipe (see figure 1) into the bottom tank during a short time period. The velocity is deduced from the volume flow and the inner cross section area of the PVC pipe. This comparison is shown in figure 7. The comparison shows that for the contrast agent, where the density of the particles is approximately the same as that of the liquid, the two methods give similar results. For the Magnetite measurements we note that the measured particle velocities are in average higher than the reference flow, because the particles are heavier than the liquid, and because of

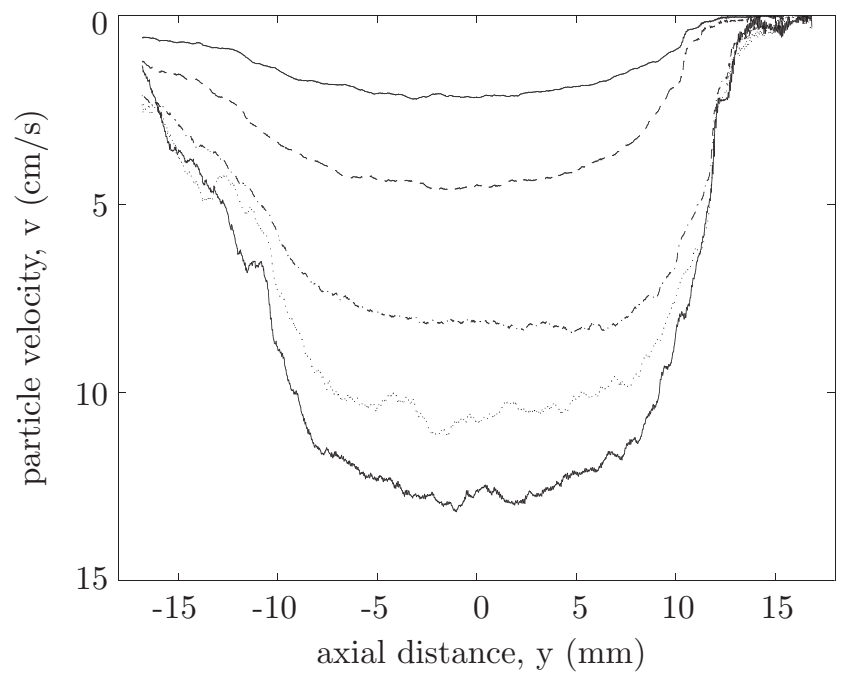

Fig. 4. Velocity profiles for Sonazoid at the center transducer element, corresponding to five values of the volume flow velocity (from top to bottom in the figure): $1.49 \mathrm{~cm} / \mathrm{s}, 2.96 \mathrm{~cm} / \mathrm{s}, 5.72$ $\mathrm{cm} / \mathrm{s}, 7.93 \mathrm{~cm} / \mathrm{s}$, and $8.96 \mathrm{~cm} / \mathrm{s}$. The hole in the pipe, where the array was mounted, was to the left in the plot. (a)

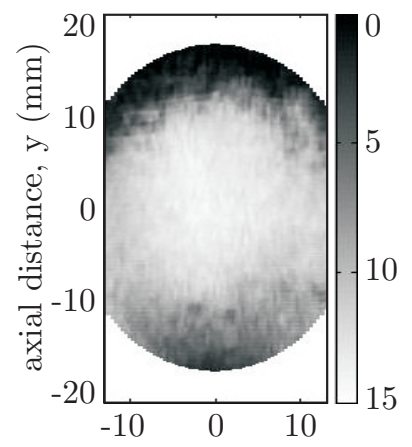

transversal distance, $\mathrm{x}(\mathrm{mm})$ (b)

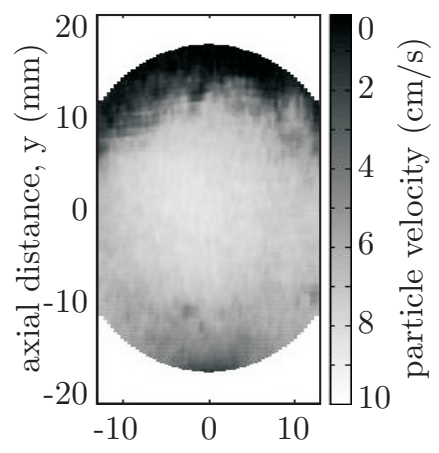

transversal distance, $\mathrm{x}(\mathrm{mm})$
Fig. 5. Magnetite particles velocity profiles for entire cross section of the flow. (a) volume flow velocity $=8.96 \mathrm{~cm} / \mathrm{s}$. (b) volume flow velocity $=5.72 \mathrm{~cm} / \mathrm{s}$. The array was located at the bottom of the images.

the vertical flow setup (moving downwards). The difference in flow velocities is therefore expected and depends on factors such as liquid viscosity, particle sizes, and solid/liquid density ratio [6].

\section{DISCUSSION}

The measurement principle presented in this paper enables us to measure displacements along the transducer axis. This can be done over the entire cross section of the pipe, which is a major improvement compared to single-transducer doppler- or correlation-based techniques. Transversal displacements, however, can not be estimated with the proposed method. The reason for this is that there is a large difference in resolution between the axial and the 


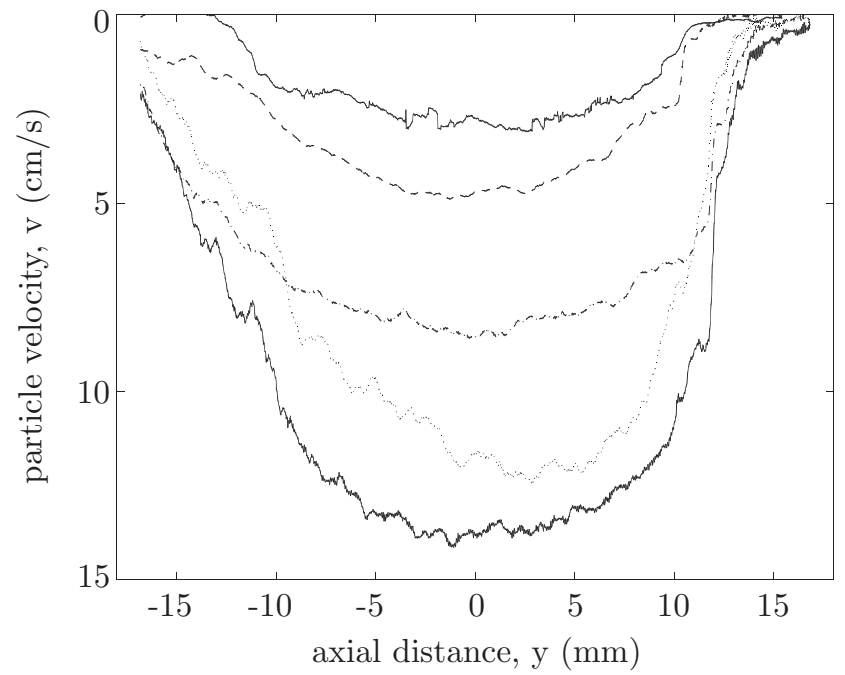

Fig. 6. Velocity profiles for Magnetite at the center transducer element, corresponding to five values of the volume flow velocity (from top to bottom in the figure): $1.49 \mathrm{~cm} / \mathrm{s}, 2.96 \mathrm{~cm} / \mathrm{s}$, $5.72 \mathrm{~cm} / \mathrm{s}, 7.93 \mathrm{~cm} / \mathrm{s}$, and $8.96 \mathrm{~cm} / \mathrm{s}$. The hole in the pipe, where the array was mounted, was to the left in the plot.

transversal direction. In the axial direction, the resolution is given by the wavelength of the sound, and the sampling time in the hardware. The measured displacements are in the range of 1-2 samples, which in the current setup corresponds to approximately $0.05 \mathrm{~mm}$. The resolution in the transversal direction, on the other hand, is given by the array pitch (i.e. spacing between array elements.), which is in our case was $0.417 \mathrm{~mm}$. With the current frame-rate, the medium will not move enough for the transversal displacement to be detectable, and if the frame-rate would be lowered for this to change, the ultrasound signals in the axial direction would be de-correlated. In a flow measurement application, the dominant motion is, however, in one direction and thus the proposed technique gives satisfying results.

For applications where the motion is slower, but 2D, one could either use two arrays mounted perpendicular to each other [5], or use the method by Bercoff, et al. [7].

\section{CONCLUSIONS}

In this paper we show how 2D USV can be used to measure particle velocity profiles in multiphase flows. This is an important step forward in flow measurement applications where the goal is to measure mass flow of the individual constituent phases. Where traditional Doppler and crosscorrelation techniques only measure the particle velocities along the axis of the transducer, the USV technique enables us to measure the particle velocity profile of an entire cross section of the flow.

Experiments on Sonazoid and Magnetite shows that the method accurately measures the particle velocities, even when liquid and solid phases move at different speeds.

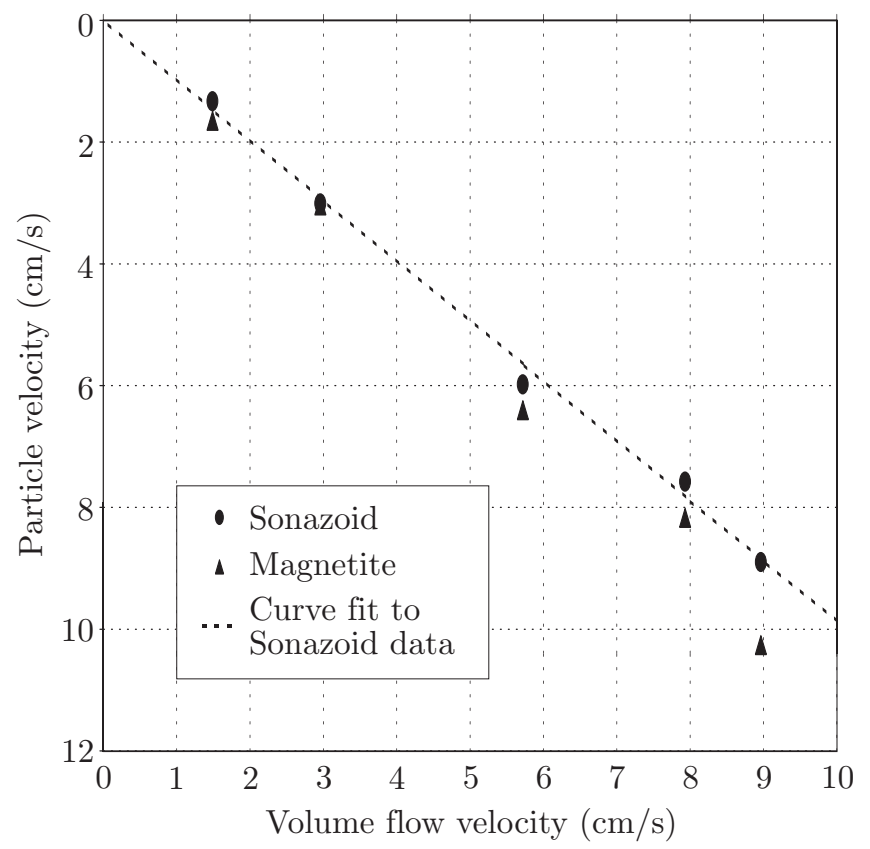

Fig. 7. Comparison with average particle velocity and total volume flow.

\section{ACKNOWLEDGEMENTS}

This work was made possible by grants from The Research Council of Norrbotten. The authors would also like to express their gratitude towards professor Mathias Fink for supporting this work, to Mr. Michel Parise for his help with the experimental setup, and to Dr. Jonny Østensen at Nycomed Amersham A/S for providing the Sonazoid contrast agent.

\section{REFERENCES}

[1] L. Sandrin, S. Manneville, and M. Fink, "Ultrafast TwoDimensional Speckle Velocimetry: A Tool In Flow Imaging," Applied Physics Letters, vol. 78, no. 8, pp. 1155-1157, 2001.

[2] J. Carlson, "Joint Measurement of Particle Distribution and Particle Mass Fraction In Multiphase Flows Using a Clamp-On PVDF Array," in Proc. of Flow Measurement 2001 (Peebles, Scotland, UK), May 7-10, 2001.

[3] J. Chaoki, L. Larachi, and M. P. Dudoković, Non-Invasive Monitoring of Multiphase Flows. Elsevier, 1997.

[4] S. Manneville, L. Sandrin, and M. Fink, "Investgating a Stretched Vortex With Ultrafast 2D Ultrasonic Speckle Velocimetry," Physics of Fluids, vol. 13, no. 6, 2001.

[5] J. Carlson, R. K. Ing, J. Bercoff, and M. Tanter, "Vortex Imaging Using Two-Dimensional Ultrasonic Speckle Correlation," in Proc. of IEEE Int. Ultrasonics Symposium 2001, vol. 1, pp. 559-562. (Atlanta, GA, USA), October 7-10, 2001.

[6] G. K. Batchelor, Introduction to Fluid Dynamics. Cambridge University Press, p. 234, 1967, Cambridge, UK.

[7] J. Bercoff, M. Tanter, S. Catheline, L. Sandrin, and M. Fink, ”Ultrafast Imaging with 2D Displacement Vector Measurements: Application to Transient Elastography and Color Flow Mapping," Proc. of Int. Ultrasonics Symposium 2001, vol. 2, pp. 1619-1622, (Atlanta, GA, USA), October 7-10, 2001. 